\title{
Concepts on Coloring of Cluster Hypergraphs with Application
}

\author{
Sovan Samanta $\left(\mathbb{D},{ }^{1}\right.$ Jeong Gon Lee $\mathbb{D}^{2},{ }^{2}$ Usman Naseem $\mathbb{D}^{3},{ }^{3}$ Shah Khalid Khan $\mathbb{D}^{4}$, \\ and Kousik Das $\mathbb{D}^{5}$ \\ ${ }^{1}$ Department of Mathematics, Tamralipta Mahavidyalaya, Tamluk, WB 721636, India \\ ${ }^{2}$ Division of Applied Mathematics, Wonkwang University, 460, Iksan-daero, Iksan-Si, Jeonbuk 54538, Republic of Korea \\ ${ }^{3}$ School of Computer Science, University of Technology Sydney, Ultimo, Australia \\ ${ }^{4}$ School of Engineering, RMIT University, Carlton, Victoria 3053, Australia \\ ${ }^{5}$ Department of Mathematics, D. J. H. School, Dantan, WB 721451, India \\ Correspondence should be addressed to Jeong Gon Lee; jukolee@wku.ac.kr
}

Received 17 April 2020; Revised 15 May 2020; Accepted 25 June 2020; Published 11 August 2020

Academic Editor: Jean Jacques Loiseau

Copyright (C) 2020 Sovan Samanta et al. This is an open access article distributed under the Creative Commons Attribution License, which permits unrestricted use, distribution, and reproduction in any medium, provided the original work is properly cited.

Coloring of graph theory is widely used in different fields like the map coloring, traffic light problems, etc. Hypergraphs are an extension of graph theory where edges contain single or multiple vertices. This study analyzes cluster hypergraphs where cluster vertices too contain simple vertices. Coloring of cluster networks where composite/cluster vertices exist is done using the concept of coloring of cluster hypergraphs. Proper coloring and strong coloring of cluster hypergraphs have been defined. Along with these, local coloring in cluster hypergraphs is also provided. Such a cluster network, COVID19 affected network, is assumed and colored to visualize the affected regions properly.

\section{Introduction}

A hypergraph is a generalization of a graph in which any subset of a vertex set is an edge rather than two vertex sets. Specially, Berge [1-3] introduced hypergraphs as generalization of graph theory. Burosch and Cecherini [4] characterized cube-hypergraphs, where each hyperedge contains three vertices. Sonntag and Teichert $[5,6]$ defined hypertrees, and they extended the notion to competition hypergraphs in another paper in 2004. To capture the notion of a cluster node, cluster hypergraphs have been introduced by Samanta et al. [7]. Uniformity and completeness properties of cluster hypergraphs have been developed here.

There are two common ways of defining colorings of hypergraphs. The first was introduced by Erdős and Hajnal $[8,9]$ and involves that no edge is monochromatic. Such a coloring is sometimes referred to as weak coloring. In the other case, strong coloring/rainbow coloring is done, in such a way that no two vertices belonging to a single edge share the same color [10]. Alon and Bregman [11] introduced 2 coloring in regular and uniform hypergraphs. This work has been extended by Vishwanathan [12]. Frieze and Mubayi [13] introduced the coloring in simple hypergraphs. Chang and Lawler [14] discussed edge coloring of hypergraphs. They also solved the conjecture of Erdos, Faber, and Lovász. Furmanczyk and Obszarski [15] extended the coloring concepts to the equitable coloring of a hypergraph. Beck [16] discussed some properties on 3-chromatic hypergraphs. Obszarski and Jastrzębski [17] added more results on edge coloring of 3-uniform hypergraphs to their earlier studies [15]. Nešetřil et al. [18] defined achromatic number of simple hypergraphs. In hypergraphs, a polychromatic coloring [19] is a coloring of its vertices such that every hyperedge comprises at least one vertex of each color. A polychromatic $m$-coloring of a hypergraph resembles a cover $m$-decomposition of its dual hypergraph. Král'a et al. [20] proved that feasible sets of mixed hypertrees are gap-free. Equitable colorings of a uniform hypergraph [21] deal with an extremal problem.

Dvořák and Postle [22] presented the idea of so-called DP-coloring, thereby extending the concept of list-coloring. DP-coloring was analyzed in detail by Bernshteyn, 
Kostochka, and Pron for graphs and multigraphs. DP-degree [23] for hypergraphs has been extended, with reminiscence that "a vertex coloring of a hypergraph is called proper if there are no monochromatic edges under this coloring. A hypergraph is said to be equitably r-colorable if there is a proper coloring with $\mathbf{r}$ colors such that the sizes of any two color classes differ by at most one."

The chronological literature review is shown in Table 1 and Figure 1. All the mentioned studies focused on the vertex and edge coloring of hypergraphs. Here, the cluster node concepts have been considered for coloring of nodes. The existing coloring concepts are not adequate to this newly defined cluster hypergraph. This study implements the two ways of coloring, proper and strong coloring in cluster hypergraphs. Additionally, the coloring technique of nodes inside the cluster has been depicted. This type of coloring is termed local coloring.

Until now, hypergraphs contain only simple nodes. This representation has problems while representing cluster/ group nodes. The concept of clustering can be found as follows. Clustering algorithms [27-30] are advanced as a powerful tool to examine the massive amount of data. The focal goal of these algorithms is to categorize the data in clusters of objects, so that data in each cluster are similar based on precise criteria and data from two dissimilar clusters be different as much as possible. This study initiated the concept of cluster hypergraphs [7] with several properties. Coloring on hypergraphs is an old phenomenon. As this study presents about coloring of cluster nodes, simple nodes inside the cluster nodes get the same color. This issue has been rectified using fuzzy color. Thus, this paper introduces an entirely new idea on coloring, i.e., fuzzy coloring on crisp cluster hypergraphs. At present, representation of COVID19 affected regions is focused by researchers. This coloring technique has been implemented to such cases using coloring of cluster hypergraphs.

\section{Hypergraphs}

Definition 1. Let $U=\left\{u_{1}, u_{2}, \ldots, u_{n}\right\}$ be a finite set and let $E=\left\{e_{1}, e_{2}, \ldots, e_{m}\right\}$ be a family of subsets of $U$ such that

$$
\begin{aligned}
e_{i} & \neq \phi(i=1,2, \ldots, m), \\
\bigcup_{\{i=1\}}^{m} e_{i} & =U .
\end{aligned}
$$

The pair $(U, E)$ is called a hypergraph with vertex set $U$ and hyperedge set $E$. The elements $\left\{u_{1}, u_{2}, \ldots, u_{n}\right\}$ of $U$ are vertices of a hypergraph $H$, and the sets $\left\{e_{1}, e_{2}, \ldots, e_{m}\right\}$ are hyperedges of a hypergraph $H$.

The length of a cycle is the number of edges in it. The girth of a hypergraph is the length of the shortest cycle it contains.

A vertex coloring of a hypergraph $H$ is called proper coloring, if any hyperedge of size greater than or equal to two contains at least two vertices of different colors.

A hypergraph $H=(V, E)$ consists of a finite set $V=$ $V(H)$ of vertices and a collection $E=E(H) \subseteq P(V)$ of subsets of $V$. A strong coloring of $H$ is a map
$\Psi: V(H) \longrightarrow N$ such that whenever $u, v \in e$ for some $e \in E(H), \Psi(u) \neq \Psi(v)$. The corresponding strong chromatic number $\chi_{s}(H)$ is the least number of colors for which $G$ has a strong coloring.

By the degree of a vertex $v$, denoted by $\operatorname{deg} v$, we mean the number of edges containing $v$.

By $\Delta(H)$, we denote the maximum vertex degree in the hypergraph $H$.

\section{Cluster Hypergraphs}

Definition 2. (see [7]): Let $X$ be a nonempty set and $V_{X}$ be a subset of $P(X)$ such that $\phi \notin V_{X}$ and $X \subset V_{X}$. Now, $E$ be a multiset whose elements belong to $P(P(X))$ such that

(i) $\phi \notin E$.

(ii) For each element $e \in E$, there exists at least one element $v \in V_{X}$ such that $v \in e$.

Then, $G=\left(V_{X}, E\right)$ is said to be cluster hypergraph where $V_{X}$ is said to be vertex set and $E$ is said to be multi-hyperedge set. Throughout this paper, hyperedges are termed edges. An example of cluster hypergraph is shown in Figure 2, and its corresponding virtual representation is shown in Figure 3.

Definition 3. Let $X$ be a nonempty set and $V_{X}$ be a subset of $P^{k}(X), k=1,2,3 \ldots$, such that $\phi \notin V_{X}$ and $X \subset V_{X}$. Now, $E$ be a multiset whose elements belong to $P\left(V_{X}\right)$ such that

(i) $\phi \notin E$.

(ii) For each element $e \in E$, there exists at least one element $v \in V_{X}$ such that $v \in e$.

Then, $G=\left(V_{X}, E\right)$ is said to be k-cluster hypergraph where $V_{X}$ is said to be vertex set and $E$ is said to be multihyperedge set. Generally, for $k=1,1$-cluster hypergraphs are assumed as cluster hypergraphs.

Definition 4. The nodes which are not contained in any other cluster nodes are called maximal nodes. A simple node may be termed a maximal node if it does not belong to any other nodes. For example, in Figure 4(a), the node $g$ is a simple as well as a maximal node, and the node $\{a, b\}$ is a maximal but nonsimple node.

Definition 5. A cluster hypergraph is called a $(m, n)$-uniform cluster hypergraph if each edge of the hypergraph contains exactly $m$ nodes, and each maximal node contains $n$ simple nodes. In Figure 5, a $(2,3)$-uniform cluster hypergraph is shown.

Definition 6. A cluster hypergraph is called a cluster connected cluster hypergraph $(\mathrm{CCCH})$ if there are edges which connect only maximal cluster nodes. In Figure 6, a $\mathrm{CCCH}$ has been drawn.

Definition 7. A CCCH is called a complete cluster hypergraph if there exists an edge between any two maximal nodes. An example of a complete $\mathrm{CCCH}$ is shown in Figure 7. 
TABLE 1: Chronological contributions of the authors towards coloring of cluster hypergraphs.

\begin{tabular}{lcc}
\hline Year & Authors & Contributions \\
\hline 1961 & Berge [1-3] & Introduction of hypergraphs \\
1973 & Erdos and Lovász [9] & On 3-chromatic hypergraphs \\
1974 & Toft [24] & Coloring of hypergraphs \\
1988 & Chang and Lawler [14] & Edge coloring of hypergraphs \\
1996 & Chen and Frieze [25] & Coloring of bipartite hypergraphs \\
2005 & Agnarsson and Halldorsson [10] & Strong coloring of hypergraphs \\
2013 & Frieze and Mubayi [13] & Coloring of simple hypergraphs \\
2019 & Furmanczyk, Obszarski [15] & Equitable coloring of hypergraphs \\
2020 & Edwards and Rzążewski [26] & Complete coloring of hypergraphs \\
2020 & Samanta et al. [7] & Introduction of cluster hypergraphs \\
2020 & Samanta et al. [This paper] & Coloring of cluster hypergraphs \\
\hline
\end{tabular}

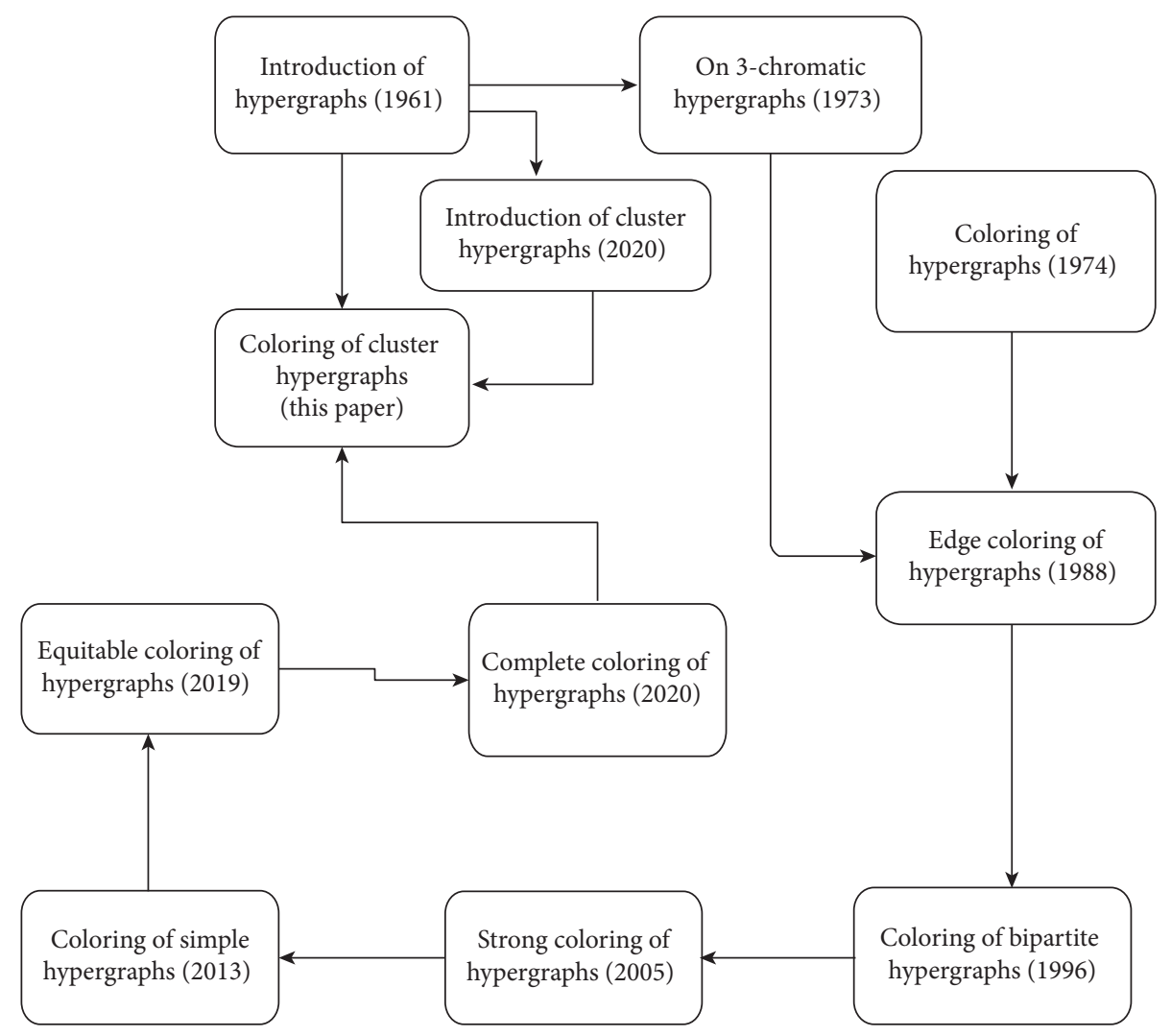

FIgURE 1: Pictorial representation of the literature of hypergraph colorings.

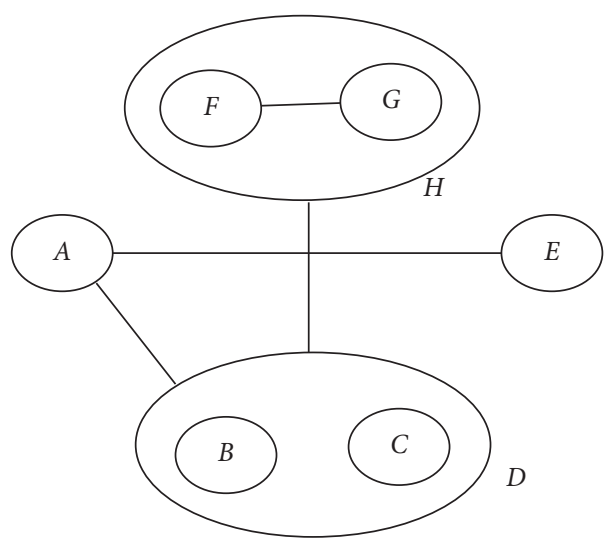

Figure 2: Cluster hypergraphs. 


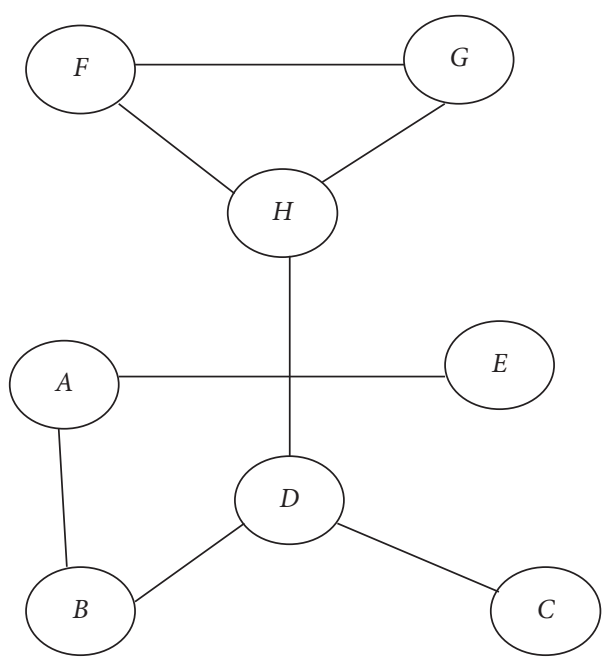

FIgURE 3: Virtual representation of Figure 2.
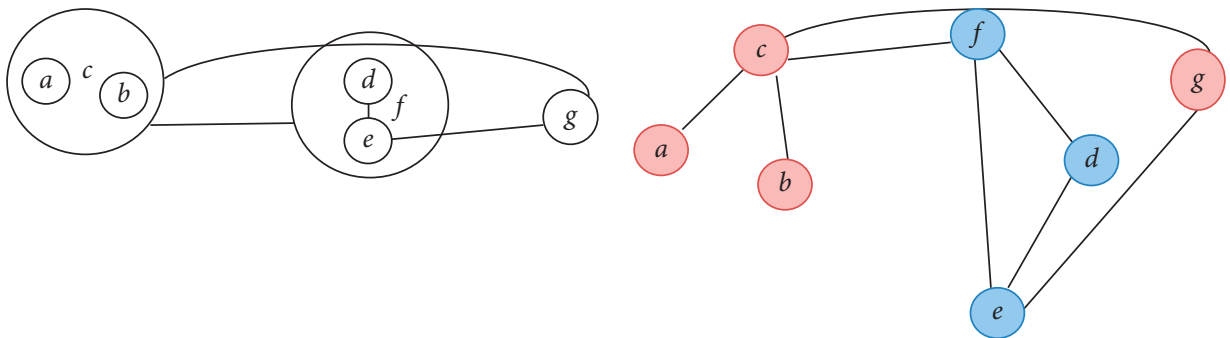

(a)

(b)

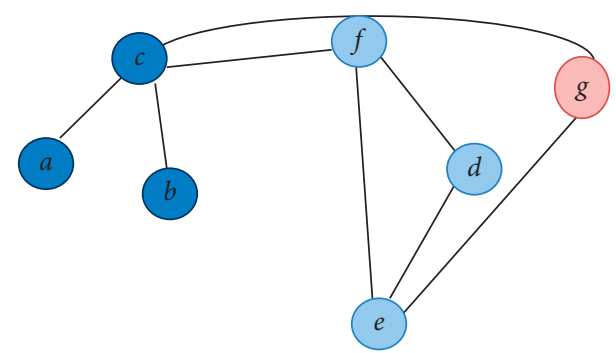

(c)

FIGURE 4: Proper and strong coloring of CCCH: (a) a cluster hypergraph; (b) proper coloring of cluster hypergraphs; (c) strong coloring of cluster hypergraphs.
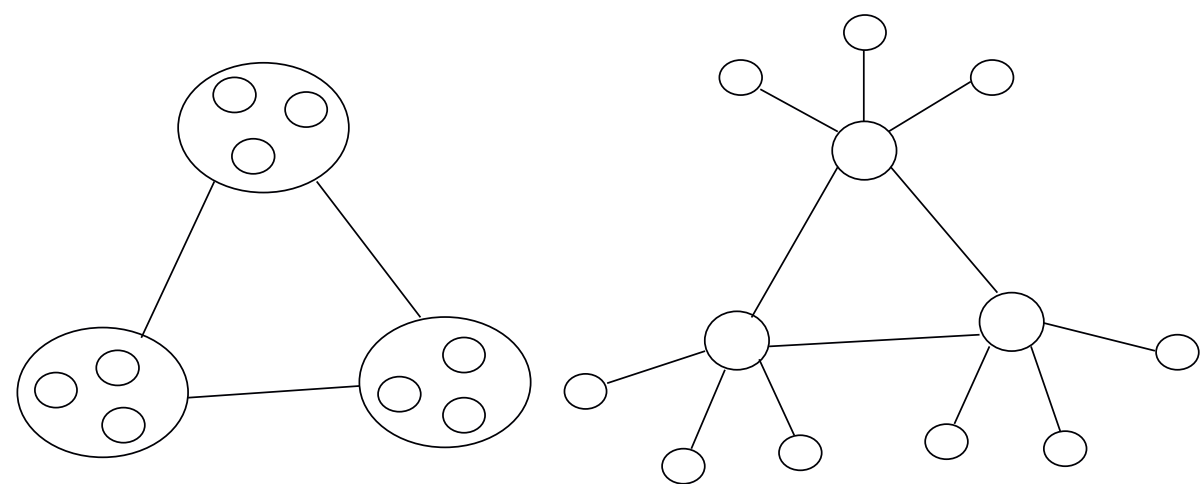

FIGURE 5: A (2, 3)-uniform cluster hypergraph. 


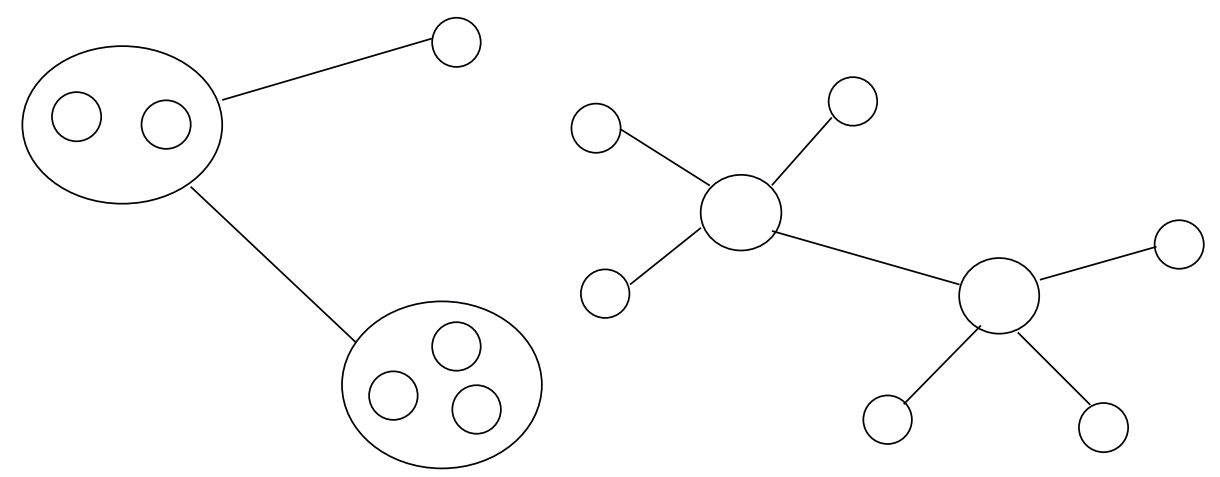

FIgURE 6: Visual representations of a CCCH.

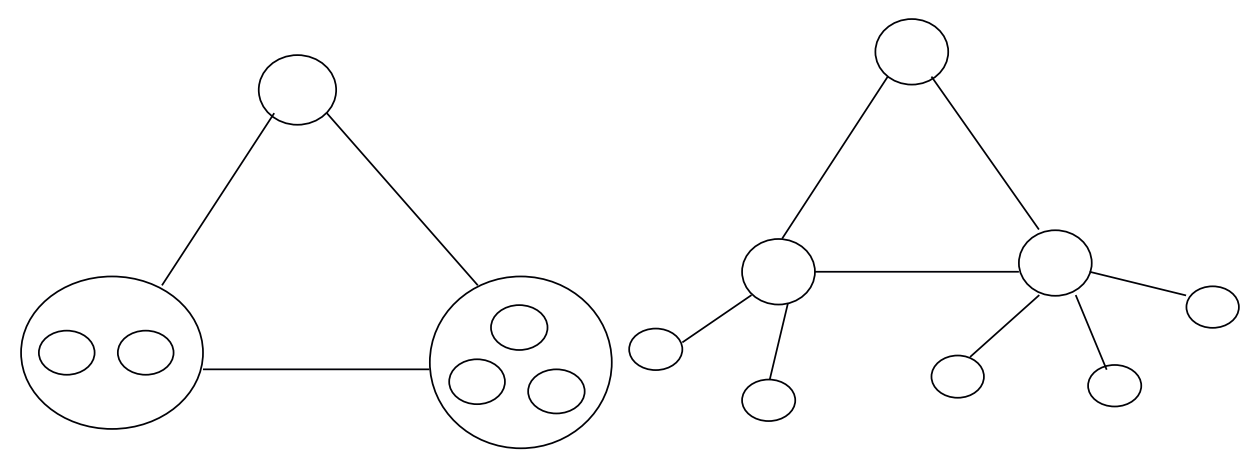

Figure 7: A complete CCCH.
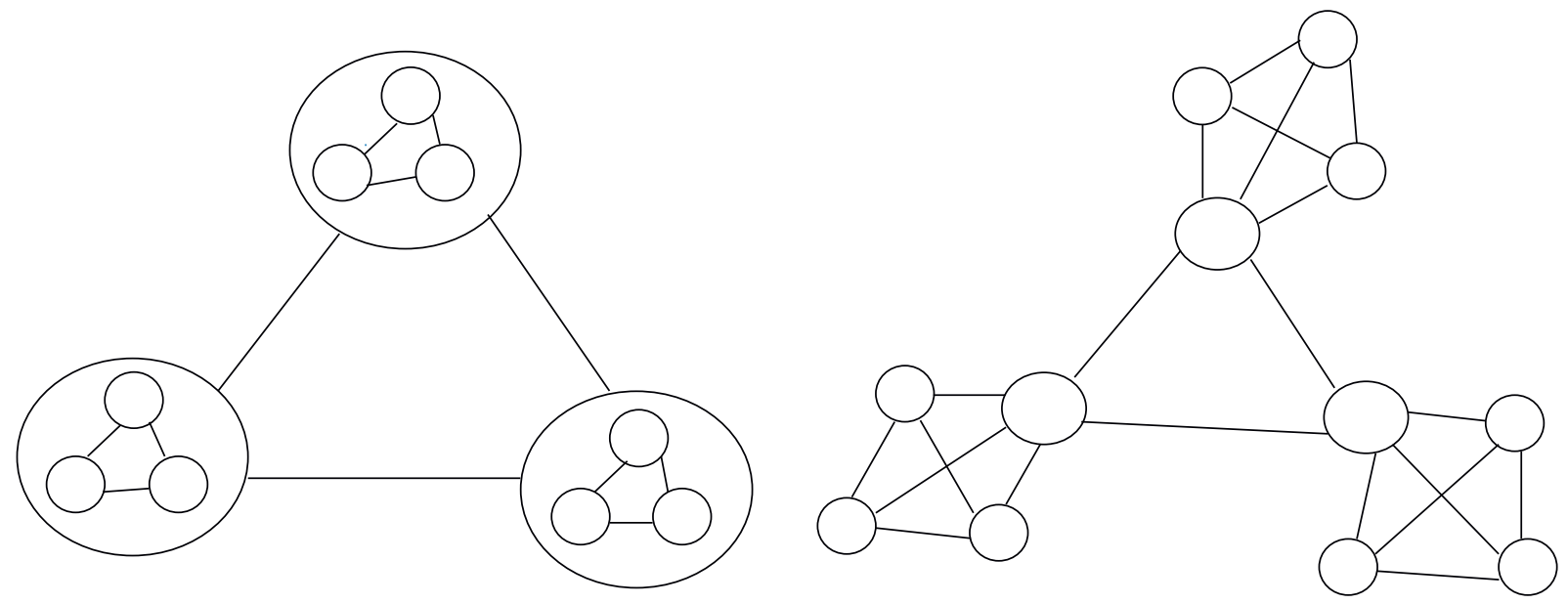

Figure 8: A complete uniform $\mathrm{CCCH}$.

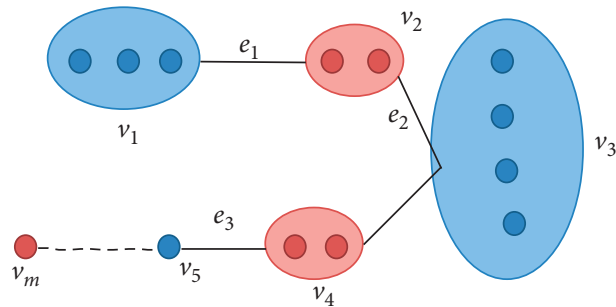

(a)

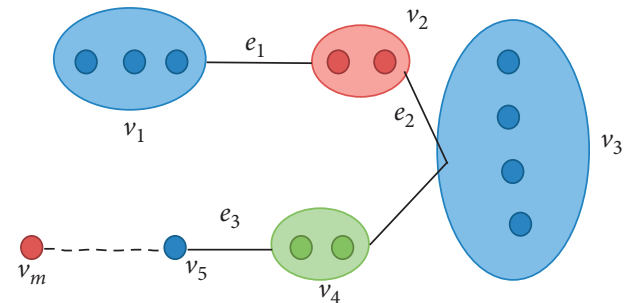

(b)

Figure 9: Coloring of paths in cluster hypergraphs: (a) coloring of a path based on proper coloring; (b) coloring of a path based on strong coloring. 


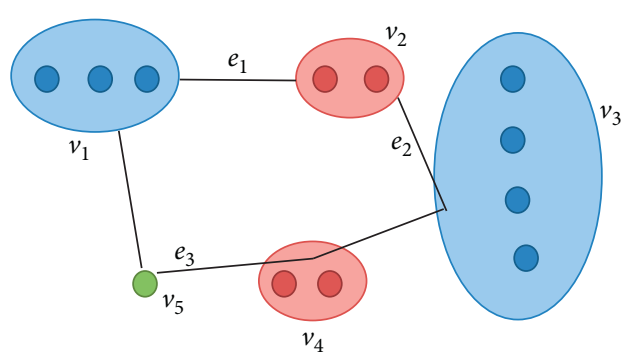

(a)

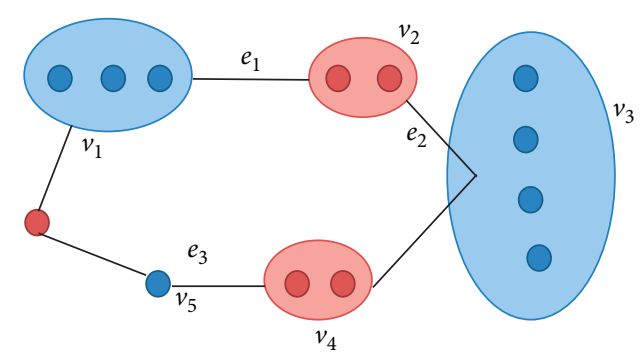

(b)

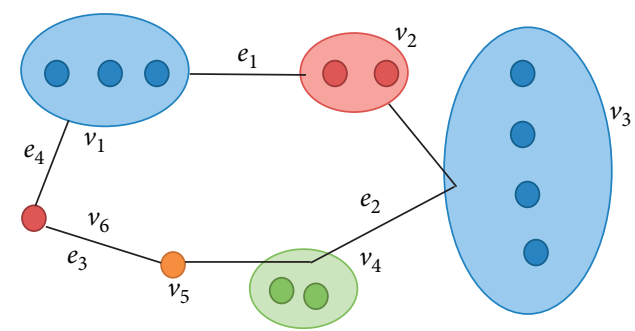

(c)

FIGURE 10: Coloring of cycles in cluster hypergraphs: (a) coloring of a cycle based on proper coloring; (b) coloring of a cycle based on proper coloring; (c) coloring of a cycle based on strong coloring.

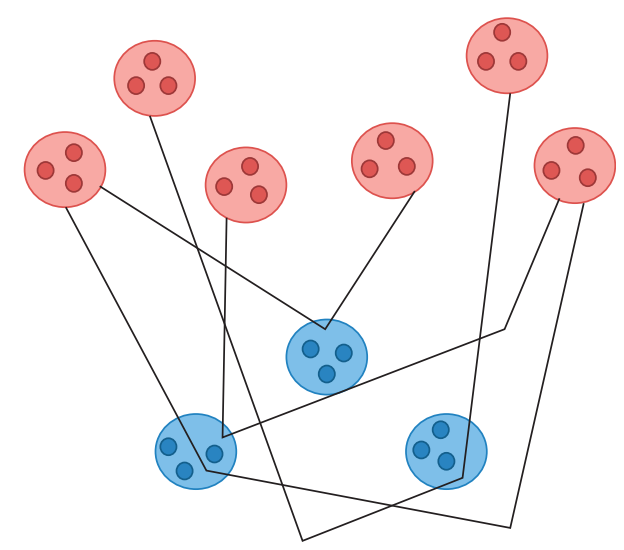

FIGURE 11: Proper coloring of 3-partite cluster hypergraphs.

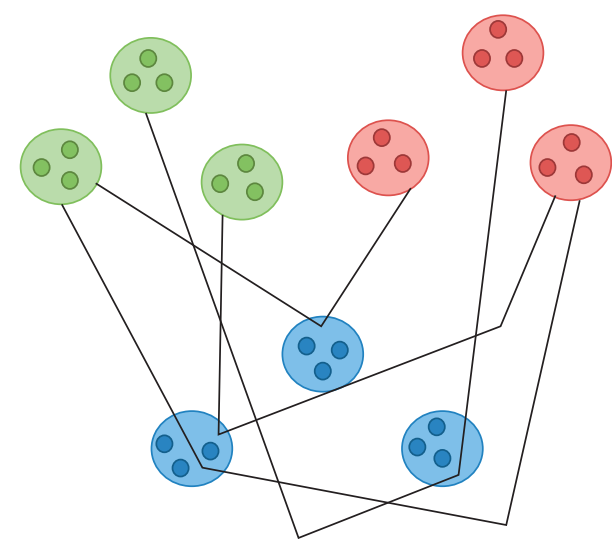

FIGURE 12: Strong coloring of 3-partite cluster hypergraphs.
Definition 8. A uniform cluster hypergraph is called a complete $(m, n)$-uniform cluster hypergraph if there exist an edge between any two maximal nodes and an edge between any two simple nodes within cluster nodes. An example of a complete $(2,3)$-uniform cluster hypergraph is shown in Figure 8.

\section{Cluster Hypergraph Coloring}

A vertex coloring of a cluster hypergraph $H$ is called proper coloring, if any edge connecting at least two maximal nodes contains at least two such maximal nodes of different colors. All the nonmaximal nodes are given the same color as the corresponding maximal nodes.

A cluster hypergraph $H=(V, E)$ consists of a finite set $V=V(H)$ of vertices and a collection $E=E(H) \subseteq P(V)$ of subsets of V. A strong coloring of $G$ is a map $\Psi: V(H) \longrightarrow N$ such that whenever $u, v$ are maximal nodes and $u, v \in e$ for some $e \in E(G), \Psi(u) \neq \Psi(v)$.

Example 1. In Figure 4(a), a cluster hypergraph $H=\left(V_{X}, E\right)$ is drawn where $X=\{a, b, d, e, g\}, V_{X}=$ $\{a, b, d, e, g, c=\{a, b\}, f=\{d, e\}\}$, and $E=\{\{\{a, b\},\{d, e\}$, $g\},\{\{a, b\},\{d, e\}\},\{d, e\},\{e, g\}\}$. In Figure 4(b), a proper coloring is shown. The graph has three maximal nodes $c=\{a, b\}, f=\{d, e\}, g$. Now the edge $\{\{a, b\},\{d, e\}, g\}$ connects all the three maximal nodes. As per the proper coloring method, two maximal nodes are to be given a different color. The nonmaximal nodes will get the same color as of its maximal nodes and similarly for others. In Figure 4(c), strong coloring has been shown. The graph has three maximal nodes $c=\{a, b\}, f=\{d, e\}, g$. Now the 


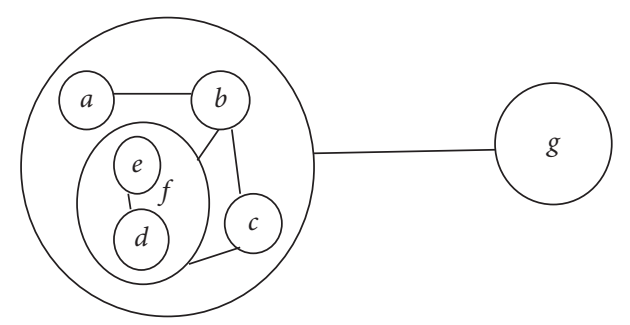

(a)

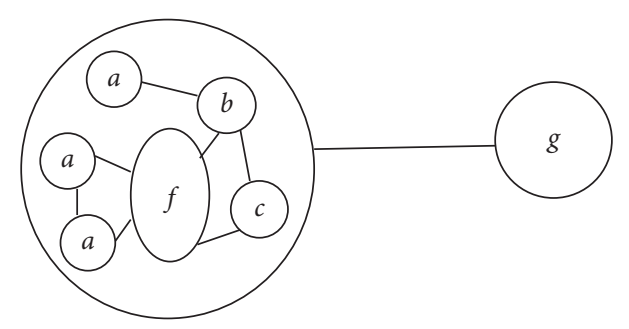

(b)

FIGURE 13: Local cluster representation of a 2-cluster hypergraph: (a) cluster hypergraph; (b) virtual representation of intramaximal nodes of (a).

TABLE 2: Local degrees of nonmaximal nodes of Figure 13.

\begin{tabular}{lcc}
\hline Vertices & Local degree & Normalized value \\
\hline$a$ & 1 & 0.25 \\
$b$ & 3 & 0.75 \\
$c$ & 2 & 0.50 \\
$d$ & 2 & 0.50 \\
$e$ & 2 & 0.50 \\
$f$ & 4 & 1.00 \\
\hline
\end{tabular}

edge $\{\{a, b\},\{d, e\}, g\}$ connects all the three maximal nodes. Hence, as per the strong coloring method, the nodes in an edge will get separate colors. The nonmaximal nodes will get equal color as of its maximal nodes and similarly for others.

4.1. Chromatic Number. The minimum number of color to use the proper coloring (or strong coloring) method for coloring cluster hypergraphs is termed chromatic number based on proper coloring (or strong coloring). The corresponding proper chromatic number $\chi(H)$ is the least number of colors for which $H$ has a proper coloring. The corresponding strong chromatic number $\chi_{s}(H)$ is the least number of colors for which $H$ has a strong coloring.

Theorem 1. The chromatic number based on strong coloring (or proper coloring) of a path in a cluster hypergraph is size of an edge containing the maximum number of maximal nodes (or two).

Proof. Let us consider a path $\left\{v_{1}, e_{1}, v_{2}, e_{2}, \ldots, v_{m}\right\}$ in a cluster hypergraph $H=\left(V_{X}, E\right)$, where $v_{i}, i=1,2, \ldots m$, are maximal nodes (see Figure 9).

Case 1 (strong coloring): As per definition of strong coloring, each node of an edge gets separate color. Thus, size of an edge containing the maximum number of maximal nodes gets maximum color. Hence, the chromatic number $\chi(H)$ based on strong coloring of a path in cluster hypergraphs is the size of an edge containing a maximum number of maximal nodes.
Case 2 (proper coloring): As per the definition of proper coloring, two nodes of an edge get separate color. Thus, in a path, maximal nodes get alternating color. The chromatic number based on proper coloring of a path in a cluster hypergraph is two.

Theorem 2. The chromatic number $\chi_{s}(H)$ based on strong coloring of a cycle in cluster hypergraphs is the size of an edge containing the maximum number of maximal nodes provided a number of such maximal nodes is greater than or equal to three; otherwise, the chromatic number is two or three.

Proof. Let us consider a cycle $\left\{v_{1}, e_{1}, v_{2}, e_{2}, \ldots, v_{m}, e_{m}, v_{1}\right\}$ in a cluster hypergraph $H=\left(V_{X}, E\right)$, where $v_{i}, i=1,2, \ldots m$, are maximal nodes (see Figure 10). Also, let the maximum size of an edge in the graph be $k$.

Case $1(\mathbf{k} \geq 3)$ : As per the definition of strong coloring, each node of an edge gets separate color. Thus, the size of an edge containing the maximum number of maximal nodes gets maximum color. Hence, the chromatic number based on strong coloring of a cycle in a cluster hypergraph is size of an edge containing the maximum number of maximal nodes.

Case $2(\mathbf{k}=2)$ : As the cluster hypergraph contains edges of size two, for odd length cycles, the chromatic number is three, and for even length cycles, the chromatic number is two.

Corollary 1. The chromatic number based on proper coloring of a cycle in cluster hypergraphs is two provided the maximum size of an edge is greater than or equal to three. If the maximum size of an edge is equal to two, even length cycles have chromatic number two and three for odd length cycles.

Theorem 3. Chromatic number of a CCCH is at least two and at most equal to the number of maximal nodes of the graph.

Proof. Let us consider a CCCH. Now the edges may contain two maximal nodes at least. In that case, the chromatic number is the number of maximal nodes in the graph. If the edges contain more than two vertices, then the chromatic number may reduce to two, if all nodes are included in a single edge. 
TABle 3: Country-wise COVID19 affected people.

\begin{tabular}{|c|c|c|c|}
\hline No. & Country & COVID19 affected people & Total deaths \\
\hline 1 & USA & $3,11,637$ & 8,454 \\
\hline 2 & Spain & $1,30,759$ & 12,418 \\
\hline 3 & Italy & $1,24,632$ & 15,362 \\
\hline 4 & Germany & 96,108 & 1,444 \\
\hline 5 & France & 89,953 & 7,560 \\
\hline 6 & China & 81,669 & 3,329 \\
\hline 7 & Iran & 58,226 & 3,603 \\
\hline 8 & UK & 41,903 & 4,313 \\
\hline 9 & Turkey & 23,934 & 501 \\
\hline 10 & Switzerland & 20,510 & 666 \\
\hline 11 & Belgium & 19,691 & 1,447 \\
\hline 12 & Netherlands & 16,627 & 1,651 \\
\hline 13 & Canada & 14,018 & 233 \\
\hline 14 & Austria & 11,850 & 204 \\
\hline 15 & Portugal & 10,524 & 266 \\
\hline 16 & Brazil & 10,360 & 445 \\
\hline 17 & S. Korea & 10,237 & 183 \\
\hline 18 & Israel & 8,018 & 46 \\
\hline 19 & Sweden & 6,443 & 373 \\
\hline 20 & Australia & 5,687 & 34 \\
\hline 21 & Norway & 5,645 & 62 \\
\hline 22 & Russia & 4,731 & 43 \\
\hline 23 & Ireland & 4,604 & 137 \\
\hline 24 & Czechia & 4,475 & 62 \\
\hline 25 & Chile & 4,161 & 27 \\
\hline 26 & Denmark & 4,077 & 161 \\
\hline 27 & Romania & 3,864 & 148 \\
\hline 28 & Poland & 3,834 & 84 \\
\hline 29 & Malaysia & 3,662 & 61 \\
\hline 30 & India & 3,588 & 99 \\
\hline 31 & Ecuador & 3,465 & 172 \\
\hline 32 & Philippines & 3,246 & 152 \\
\hline 33 & Japan & 3,139 & 77 \\
\hline 34 & Pakistan & 2,880 & 45 \\
\hline 35 & Luxembourg & 2,729 & 31 \\
\hline 36 & Saudi Arabia & 2,370 & 29 \\
\hline 37 & Indonesia & 2,273 & 198 \\
\hline 38 & Thailand & 2,169 & 23 \\
\hline 39 & Finland & 1,927 & 25 \\
\hline 40 & Mexico & 1,890 & 79 \\
\hline 41 & Panama & 1,801 & 46 \\
\hline 42 & Peru & 1,746 & 73 \\
\hline 43 & Greece & 1,673 & 68 \\
\hline 44 & Serbia & 1,624 & 44 \\
\hline 45 & South Africa & 1,585 & 9 \\
\hline 46 & D. Republic & 1,578 & 77 \\
\hline 47 & UAE & 1,505 & 10 \\
\hline 48 & Argentina & 1,451 & 43 \\
\hline 49 & Iceland & 1,417 & 4 \\
\hline 50 & Colombia & 1,406 & 32 \\
\hline 51 & Qatar & 1,325 & 3 \\
\hline 52 & Algeria & 1,251 & 130 \\
\hline 53 & Ukraine & 1,251 & 32 \\
\hline 54 & Singapore & 1,189 & 6 \\
\hline 55 & Croatia & 1,126 & 12 \\
\hline
\end{tabular}

4.2. Bipartite or 2-Colorable Cluster Hypergraphs. A cluster hypergraph $H$ is bipartite if its maximal node set can be partitioned into two independent sets (nonadjacent node set) such that every edge connects both partite sets of maximal nodes or connects intercluster nodes. Equivalently, $H$ is a bipartite if it is 2-colorable by proper coloring.

4.3. $n$-Partite Cluster Hypergraphs. Let us define a n-partite cluster hypergraph as a $n$-uniform cluster hypergraph whose maximal nodes can be partitioned into $n$ independent sets (nonadjacent node sets) and each edge is incident to exactly one maximal node from each partition or is incident to intramaximal cluster nodes only. It is to be mentioned that if there is an edge in a maximal cluster, the edge may contain any number of nodes, not necessarily $n$ nodes.

Example 2. In Figure 11, a (3,3)-uniform cluster hypergraph has been shown. It can be noted that edges connect three maximal nodes of three independent sets. Also, edges may connect intercluster nodes. Naturally, the chromatic number is 2 for this case. In Figure 12, another $(3,3)$-uniform cluster hypergraph is colored by strong coloring technique. The chromatic number $\chi_{s}(H)$ is 3 .

Note 1: Let $H$ be a cluster hypergraph of maximal vertex degree $\Delta$ of maximal nodes, such that its each edge contains at least $\boldsymbol{\delta}$ maximal nodes. Let $k=[\{2 \Delta / \delta\}]$. Then, the following holds.

(1) The chromatic number of $H$ is $k+1$ by proper vertex coloring.

(2) The chromatic number of $H$ is $k$ by proper vertex coloring if $\delta \geq 3$ and $k \geq 3$.

Note 2: Every minimally nonbipartite cluster hypergraph has at least as many edges as vertices.

Theorem 4. For any n-uniform cluster hypergraph $H$, the two inequalities hold: $\Delta(H) \leq \chi(H) \leq n \Delta(H)-n+1$.

Proof. The first inequality is true by observation. The second inequality can be acceptable in the following way. Each edge in any $n$-uniform cluster hypergraph has at most $n \Delta(H)-n$ neighbors of maximal nodes. Hence, for a certain edge $e$ even if all the adjacent edges have various colors, the algorithm has always generalized to chromatic number $\chi(H)$ of $H$ which is less than or equal to the number of neighbors +1 . Thus, $\Delta(H) \leq \chi(H) \leq n \Delta(H)-n+1$.

4.4. Local Coloring on Nonmaximal Nodes. In proper coloring or strong coloring, maximal nodes are given colors. But simple or cluster nodes inside maximal nodes are given the same color as of their corresponding maximal nodes. To differ the color of nonmaximal nodes, local degree is assumed. Local degree of a nonmaximal node is the number of intramaximal node edges incident to the node.

The local degree of a vertex $f$ in Figure 13 is 4 . The local degree of nonmaximal nodes in Figure 13 is given in Table 2.

Based on the normalized value of local degree, fuzzy colors are given to the nonmaximal nodes. If a maximal node gets a color say "BLUE", then the nodes within the maximal node with normalized value $w$ get mixed of the color BLUE $100 w \%$ and white $(100-100 w) \%$. As per Table $2, f$ gets the 


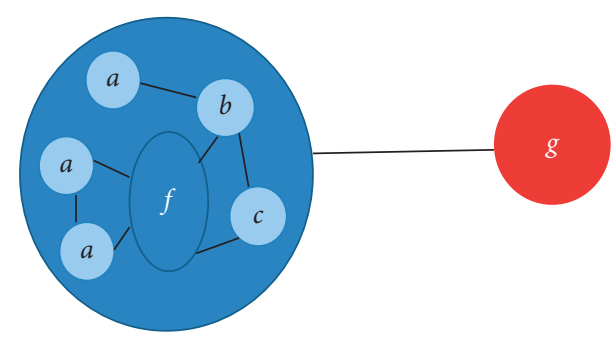

FIgURE 14: Coloring of nonmaximal nodes.

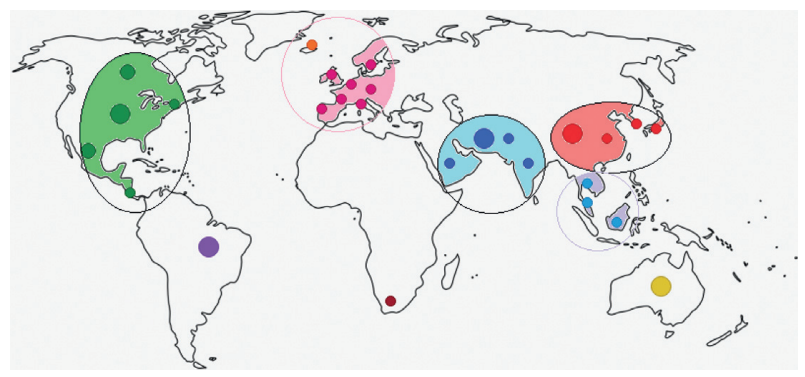

Figure 15: COVID19 affected countries.

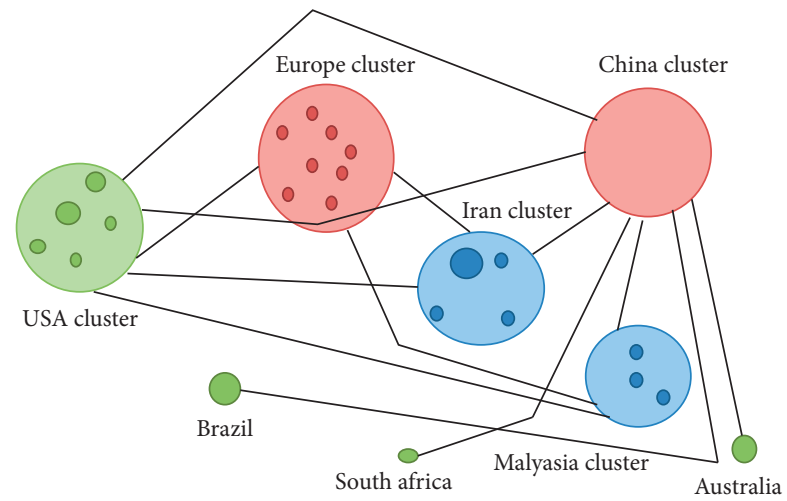

FIgURE 16: A proper coloring of the assumed network shown in Figure 15.

basic color BLUE, and $b$ gets a mixture of 75\% BLUE and $25 \%$ of white color. $c$, $d$, and $e$ get a mixture of $50 \%$ BLUE and $50 \%$ white and so on (see Figure 14).

\section{Scope of Applications}

Cluster hypergraphs are convenient to represent any social networks. The recent literature on social network analysis can be found in [31-36]. This small example illustrates the network spread by COVID19 in worldwide. The taken data are shown in Table 3. It can be noted that nearby countries (based on a certain distance) form a cluster. If one cluster is affected by other clusters, then one edge is used to connect the clusters. The network is shown in Figure 15. The network as a cluster hypergraph is colored by proper coloring (see Figure 16).

Benefits of the study are as follows:

(1) This study analyzed cluster hypergraphs, a generalized class of hypergraphs.
(2) This study provided several properties of cluster hypergraphs, including a $\mathrm{CCCH}$, a complete $\mathrm{CCCH}$, and a uniform $\mathrm{CCCH}$.

(3) Proper coloring and strong coloring have been defined for cluster hypergraphs. Local coloring for nonmaximal nodes has also been introduced.

(4) Proper coloring of COVID19 affected regions has been shown.

\section{Conclusions}

This study developed basic terminologies of cluster hypergraphs. Coloring of cluster hypergraphs are analyzed by two basic techniques. Proper coloring and strong coloring are two ways of coloring of cluster hypergraphs. Additionally, the coloring of intranodes of a maximal node is depicted. Finally, a small network is considered for the coloring purpose.

In future, several complex network problems can be solved by cluster hypergraph coloring/labelling. In particular, every social network is a cluster hypergraph. The centrality of a simple node is no longer important compared to the centrality of a cluster node. This study is a backbone for such applications.

\section{Data Availability}

The data in Table 3 have been collected from https://www. worldometers.info/coronavirus/ dated 05.04.2020. The data are available in the public domain.

\section{Conflicts of Interest}

The authors declare that they have no conflicts of interest.

\section{Acknowledgments}

This research was supported by the Basic Science Research Program through the National Research Foundation of Korea (NRF) funded by the Ministry of Education (2018R1D1A1B07049321).

\section{References}

[1] C. Berge, Fárbung Von Graphen Deren Sämtliche Bzw. Ungerade Krense Starr Sind Math. Nat. Reihe, Martin-LutherUniversity Halle-Wittenberg, Halle, Germany, 1961.

[2] C. Berge, Graphs and Hypergraphs, Elsevier, Amsterdam, The Netherlands, 1973.

[3] C. Berge, Hypergraphs, North-Holland, Amsterdam, The Netherlands, 1989.

[4] G. Burosch and P. V. Cecherini, "A characterization of cubehypergraphs," Discrete Mathematics, vol. 152, no. 1-3, pp. 55-68, 1996.

[5] M. Sonntag and H.-M. Teichert, "Sum numbers of hypertrees," Discrete Mathematics, vol. 214, no. 1-3, pp. 285-290, 2000.

[6] M. Sonntag and H.-M. Teichert, "Competition hypergraphs," Discrete Applied Mathematics, vol. 143, no. 1-3, pp. 324-329, 2004. 
[7] S. Samanta, G. Muhiudin, A. M. Alanazi, and K. Das, “A mathematical approach on representations of competitions: competition cluster hypergraphs," Mathematical Problems in Engineering, vol. 2020, Article ID 2517415, 10 pages, 2020.

[8] P. Erdős and A. Hajnal, "On chromatic number of graphs and set-systems," Acta Mathematica Hungarica, vol. 17, pp. 61-99, 1966.

[9] P. Erdos and L. Lovász, "Problems and results on 3-chromatic hypergraphs and some related questions," Colloquia Mathematica Societatis, vol. 10, pp. 609-627, 1973.

[10] G. Agnarsson and M. Halldórsson, "Strong colorings of hypergraphs," in Proceedings of the 1st Workshop on Approximation and Online Algorithms, Bergen, Norway, September 2004.

[11] N. Alon and Z. Bregman, "Every 8-uniform 8-regular hypergraph is 2-colorable," Graphs and Combinatorics, vol. 4, no. 1 , pp. $303-306,1988$.

[12] S. Vishwanathan, "On 2-coloring certain k-uniform hypergraphs," Journal of Combinatorial Theory, Series A, vol. 101, no. 1, pp. 168-172, 2003.

[13] A. Frieze and D. Mubayi, "Coloring simple hypergraphs," Journal of Combinatorial Theory, Series B, vol. 103, no. 6, pp. 767-794, 2013.

[14] W. I. Chang and E. L. Lawler, "Edge coloring of hypergraphs and a conjecture of ErdÖs, Faber, Lovász," Combinatorica, vol. 8, no. 3, pp. 293-295, 1988.

[15] H. Furmanczyk and P. Obszarski, "Equitable coloring of hypergraphs," Discrete Applied Mathematics, vol. 261, pp. 186-192, 2019.

[16] J. Beck, "On 3-chromatic hypergraphs," Discrete Mathematics, vol. 24, no. 2, pp. 127-137, 1978.

[17] P. Obszarski and A. Jastrzębski, "Edge-coloring of 3-uniform hypergraphs," Discrete Applied Mathematics, vol. 217, pp. 48-52, 2017.

[18] J. Nešetřil, K. T. Phelps, and V. Rödl, "On the achromatic number of simple hypergraphs," Ars Combinatoria, vol. 16, pp. 95-102, 1983.

[19] T. Li and X. Zhang, "Polychromatic colorings and cover decompositions of hypergraphs," Applied Mathematics and Computation, vol. 339, no. 15, pp. 153-157, 2018.

[20] D. Král'a, J. Kratochvíl, A. Proskurowski, and H. Vossc, "Coloring mixed hypertrees," Discrete Applied Mathematics, vol. 154, pp. 660-672, 2006.

[21] M. B. Akhmejanova and D. A. Shabanov, "Equitable colorings of hypergraphs with few edges," Discrete Applied Mathematics, vol. 276, pp. 2-12, 2020.

[22] Z. Dvořák and L. Postle, "Correspondence coloring and its application to list-coloring planar graphs without cycles of lengths 4 to 8," Journal of Combinatorial Theory, Series B, vol. 129 , pp. $38-54,2018$.

[23] A. Bernshteyn and A. Kostochka, "DP-colorings of hypergraphs," European Journal of Combinatorics, vol. 78, pp. 134-146, 2019.

[24] B. Toft, "Color-critical graphs and hypergraphs," Journal of Combinatorial Theory, Series B, vol. 16, no. 2, pp. 145-161, 1974.

[25] H. Chen and A. Frieze, "Coloring bipartite hypergraphs," in Lecture Notes in Computer Science, vol. 1084, pp. 345-358, Springer, Berlin, Germany, 1996.

[26] K. Edwards and P. Rzążewski, "Complete colourings of hypergraphs," Discrete Mathematics, vol. 343, no. 2, p. 111673 , 2020.

[27] A. S. Rostami, M. Badkoobe, F. Mohanna, A. A. R. Hosseinbadi, and V. E. Balas, "Imperialist competition based clustering algorithm to improve the lifetime of wireless sensor network," in Soft Computing Applications. SOFA 2016, Advances in Intelligent Systems and Computing, V. Balas, L. Jain, and M. Balas, Eds., p. 633, Springer, Berlin, Germany, 2018.

[28] A. S. Rostami, M. Badkoobe, F. Mohanna, H. Keshavarz, A. A. R. Hosseinabadi, and A. K. Sangaiah, "Survey on clustering in heterogeneous and homogeneous wireless sensor networks," The Journal of Supercomputing, vol. 74, no. 1, pp. 277-323, 2018.

[29] B. Saemi, A. A. R. Hosseinbadi, M. Kardgar, V. E. Balas, and H. Ebadi, "Nature inspired partitioning clustering algorithms: a review and analysis," in Soft Computing Applications. SOFA 2016, Advances in Intelligent Systems and Computing, V. Balas, L. Jain, and M. Balas, Eds., p. 634, Springer, Berlin, Germany, 2018.

[30] S. M. Bozorgi, A. Shokouhi Rostami, A. A. R. Hosseinabadi, and V. E. Balas, "A new clustering protocol for energy harvesting-wireless sensor networks," Computers \& Electrical Engineering, vol. 64, pp. 233-247, 2017.

[31] U. Naseem and K. Musial, "Dice: deep intelligent contextual embedding for twitter sentiment analysis," in Proceedings of the International Conference on Document Analysis and Recognition (ICDAR), Sydney, Australia, April 2019.

[32] U. Naseem, S. K. Khan, I. Razzak, and I. A. Hameed, "Hybrid words representation for airlines sentiment analysis," in Proceedings of the Australasian Joint Conference on Artificial Intelligence, p. 381-392, Adelaide, SA, Australia, December 2019.

[33] S. K. Khan, M. Farasat, U. Naseem, and F. Ali, "Performance evaluation of next-generation wireless (5G) UAV relay," Wireless Personal Communications, vol. 113, pp. 1-16, 2020.

[34] U. Naseem, I. Razzak, and I. A. Hameed, "Deep context-aware embedding for abusive and hate speech detection on Twitter," Australian Journal of Intelligent Information Processing Systems, vol. 15, no. 3, pp. 69-76, 2019.

[35] U. Naseem, I. Razzak, K. Musial, and M. Imran, "Transformer based deep intelligent contextual embedding for twitter sentiment analysis," Future Generation Computer Systems, vol. 113, pp. 58-69, 2020.

[36] K. Das, S. Samanta, and M. Pal, "Study on centrality measures in social networks: a survey," Social Network Analysis and Mining, vol. 8, no. 13, 2018. 